\title{
Prácticas adoptadas de la ISO/IEC 20000 en pequeñas organizaciones desarrolladoras de software que ofrecen mesa de servicios. Un estudio de caso
}

\author{
Gloria Baños ${ }^{1}$, Karin Melendez², Abraham Dávila ${ }^{3}$ \\ gloria.banosd@pucp.edu.pe, kmelendez@pucp.edu.pe, abraham.davila@pucp.edu.pe \\ ${ }^{1}$ Facultad de Ciencias e Ingeniería, Pontificia Universidad Católica del Perú, Lima 32, Lima, Perú \\ ${ }^{2}$ Departamento de Ingeniería, Pontificia Universidad Católica del Perú, Lima 32, Lima, Perú \\ ${ }^{3}$ Departamento de Ingeniería, Pontificia Universidad Católica del Perú, Lima 32, Lima, Perú
}

DOI: 10.17013/risti.20.1-17

\begin{abstract}
Resumen: La adecuada gestión de los servicios de las tecnologías de información influye de manera significativa en las operaciones y la calidad del servicio de las organizaciones, en particular en las que desarrollan software y ofrecen servicios asociados. Sin embargo, a pesar del despliegue de la ISO/IEC 20000 a nivel internacional, las pequeñas organizaciones que desarrollan software y ofrecen servicios en Lima no han tenido mucho progreso en su adopción. El objetivo de este estudio es determinar el grado de adopción de las prácticas de gestión de servicios de tecnología de información en pequeñas organizaciones desarrolladoras de software que ofrecen servicios asociados. El análisis se realizó usando un estudio de caso en tres pequeñas organizaciones. Se estableció que el 31\% de prácticas base de la ISO/IEC 20000-4 están adoptadas, 23\% no están adaptadas y para el resto no es posible determinar su adopción. Las prácticas adoptadas son principalmente las relacionadas a los requisitos del servicio y las incidencias; los que menos son adoptadas están relacionadas a gestión de cambio o gestión de la configuración, lo cual tiene sentido pues las organizaciones de manera natural tienden a resolver primero sus necesidades inmediatas
\end{abstract}

Palabras-clave: gestión de servicios de tecnología de información; ISO/IEC 20000; desarrollo de software; pequeñas organizaciones.

ISO/IEC 2000 practices adopted in small software development organizations that offer services desk. A case study

Abstract: An adequate perform of information technology service management has great impact in the service quality and operations that organizations provide; in special in software development organization that offer services desk. However, despite the deployment of ISO/IEC 20000 in the international context, small organizations that develop software and provide services in Lima have not had much progress in adoption. The aim of this study is to determine the practices adoption 
from information technology service management in small software development organizations that offer services desk. Analysis was performed using a case study in three small organizations. It was established that $31 \%$ of practices based on ISO/IEC 20000-4 are adopted, 23\% are not adapted and the rest is not possible to determine their adoption. The adopted practices are mainly related to incidents and service requirements; and the least adopted are related to change management and configuration management, this situation is aligned with naturally trend to first solve their immediate needs

Keywords: Information Technology Service Management, ISO/IEC 200oo, software development, small organization.

\section{Introducción}

Las organizaciones desarrolladoras de software buscan aumentar la competitividad y la satisfacción de los clientes para lo cual aplican modelos y estándares para la mejora de procesos de software (Mejia, Muñoz, \& Rocha, 2014). Además, están interesadas en crear buenas expectativas sobre los servicios que brindan, por lo que tienen un especial interés en la mejora de procesos para la gestión de servicios de tecnología de información (GSTI) (Mesquida, Mas, San, \& Arcilla, 2014). La GSTI cumple un rol determinante en las organizaciones, pues es un aspecto relevante para el soporte a las operaciones y las decisiones (Arcilla, Calvo-Manzano, \& San Feliu, 2013; Rocha \& Freixo, 2015); por lo que las propuestas como ITIL (ITIL, 2015), CMMI-SRV (SEI, 2010) o ISO/IEC 20000 (ISO, 2011) han tenido gran aceptación a nivel internacional. Sin embargo, en el contexto de las pequeñas empresa (u organizaciones en general), algunos estudios ( (Göbel, Cronholm, \& Seigerroth, 2013), (Küller, Vogt, Hertweck, \& Grabowski, 2012), (Machado, Reinehr, \& Malucelli, 2012)) señalan que existe una brecha entre el conocimiento de los marcos de referencia de la GSTI y su implementación. Asimismo, en Küller et al. (Küller, Vogt, Hertweck, \& Grabowski, 2012), se señala que el 52\% de las empresas pequeñas de Europa conocen de los modelos de gestión de servicios, sin embargo, sólo lo aplican el 10\%. Finalmente, para varios autores como Göbel et al. (Göbel, Cronholm, \& Seigerroth, 2013), Küller et al. (Küller, Vogt, Hertweck, \& Grabowski, 2012), Machado et al. (Machado, Reinehr, \& Malucelli, 2012), Hoerbst et al. (Hoerbst, Hackl, Blomer, \& Ammenwerth, 2011) y Qi (Qi, 2011), los principales problemas con estos marcos de trabajo de GSTI son la complejidad de los modelos, la falta de conocimiento y la ausencia de guías adecuadas para su adopción.

De otro lado, a partir del Proyecto Competisoft, componente Perú, según Dávila et al. (Dávila, y otros, 2012), se pudo establecer que un grupo importante de pequeñas empresas participantes de dicho Proyecto realizaban actividades de desarrollo de software y gestión de servicios software. A partir de este hecho, el proyecto ProCal-ProSer (Dávila, 2013) definió como foco las pequeñas organizaciones (PO), y se ha decidido, entre otros, estudiar sobre la aplicación de la GSTI en PO, elaborar y probar un modelo de GSTI para una PO y determinar los factores que influyen en su adopción en una PO que desarrollan software y ofrece servicios software.

En especial, para este trabajo, se estableció la necesidad de determinar el grado de adopción de las prácticas de GSTI recogidas en la ISO/IEC 20000-4 en el contexto de las pequeñas organizaciones desarrolladoras de software y que ofrecen servicios 
software. El artículo se organiza de la siguiente manera: la Sección 2 presenta los modelos considerados; la Sección 3 presenta trabajos relacionados; la Sección 4 presenta el protocolo de la investigación seguida para este estudio; la Sección 5 muestra los resultados obtenidos y el análisis correspondiente; y la Sesión 6 presenta la discusión final y trabajo futuro.

\section{Modelos considerados en el estudio}

Este trabajo se realizó como parte del proyecto ProCal-ProSer (Dávila, 2013) por lo que se trabajó con los modelos ISO como principal referencia. En particular, para este trabajo se han tomados como base: (a) los relacionados a la GSTI en la ISO y (b) la ISO/ IEC 15504-2 que ha sido usada para la evaluación de los procesos.

\subsection{Modelos de GSTI en ISO.}

La serie de normas ISO/IEC 20000 comprende varios documentos, siendo la ISO/ IEC 20000-1 la que define los requisitos del sistema de GSTI (ISO, 2011) y la ISO/ IEC 20000-4 la que define el modelo de proceso de referencia asociado (ISO, 2010). Adicional-mente, convienen mencionar a la ISO/IEC 15504-8 (ISO, 2012) que es un modelo de evaluación de procesos para la ISO/IEC 20000-4.

La ISO/IEC 20000-1 (ISO, 2011) es un documento que contiene buenas prácticas relacionadas a la GSTI, cuya finalidad es que los proveedores prevean un servicio de calidad a sus clientes. Los requisitos del documento incluyen aspectos de diseño, transición, envío y mejora de servicios que cumplen con requisitos del servicio y que proveen valor tanto al cliente como al proveedor del servicio (ISO, 2011).

La ISO/IEC 20000-4 (ISO, 2010) es un documento que contiene el modelo de procesos de referencia (PRM de Process Reference Model) para la GSTI, que describe los elementos de los procesos del sistema de gestión de servicios de la ISO/IEC 20000-1. Cada proceso en la ISO/IEC 20000-4 está descrito en términos de propósitos y resultados, siendo estos últimos los mínimos necesarios para cumplir con los requisitos de la ISO/IEC 20000-1 (ISO, 2010). Asimismo, la ISO/IEC 20000-4 permite el desarrollo del modelo de evaluación de procesos (PAM de Process Assessment Model) para la GSTI y que es lo que se presenta en la ISO/IEC 15504-8 (ISO, 2012). Este PAM presenta un conjunto de indicadores usados para evaluar el desempeño y capacidad de los procesos de la ISO/ IEC 20000-4, obtenidos y para desarrollar un programa de mejora (ISO, 2012).

\subsection{Modelo de evaluación de procesos ISO/IEC 15504-2}

La ISO/IEC 15504-2 (ISO, 2003) es un estándar para la evaluación de procesos que establece los requisitos para evaluar los PRM, que en nuestro caso será la ISO/IEC 20000-4 (ISO, 2010). A partir del marco de mediciones que tiene la ISO/IEC 15504-2 (ISO, 2003) y en conjunto con el PRM se define un PAM que en nuestro caso sería la ISO/IEC 15504-8 (ISO, 2012). Sobre la ISO/IEC 15504-2 se ha realizado un estudio (Simon, Emam, Rousseau, Jacquet, \& Babey, 1997) que demuestra su alta fiabilidad en la evaluación de procesos, lo que representa una fortaleza para nuestro trabajo. Además, la ISO/IEC 15504-2 (ISO, 2003) establece: (i) las capacidades de proceso, desde un 
nivel o hasta un nivel 5, caracterizados por los atributos de proceso; (ii) un conjunto de atributos de proceso, que comienzan en AP.1.1 que denota un proceso realizado y que es correspondiente con el primer nivel de capacidad; y para los siguiente niveles se usa dos atributos de proceso para cada nivel de capacidad; y (iii) una escala de calificación de los atributos de proceso en relación al logro del propósito del proceso: $\mathrm{N}$ (no logrado), $\mathrm{P}$ (parcialmente logrado), A (ampliamente logrado) y T (totalmente logrado).

\section{Trabajos relacionados}

Algunas organizaciones han realizado adopciones parciales sobre modelos de GSTI (sólo para ciertos procesos en función de sus problemas o necesidades de mejora), desarrollando modelos propios según sus requisitos y utilizando modelos como: ITIL®2011, CMMI-SVC, COBIT®5 e ISO/IEC 20000 (Melendez \& Dávila, 2015).

En el trabajo de Liu, Gao, Luo y Wan (Liu, Gao, Luo, \& Wan, 2011) la organización implementó la gestión de servicios de TI desarrollando un proyecto de mejora en el cual agrupó los procesos deseados en tres fases. Los procesos incluidos en la primera fase fueron: gestión de incidentes y gestión de problemas; la segunda fase fue: gestión de nivel de servicio, costos y cambios; y en la tercera fase: gestión de la continuidad del servicio, entre otros (Liu, Gao, Luo, \& Wan, 2011).

Lahtela, Jantti y Kaukola (Lahtela, J“antti, \& Kaukola, 2010) mostraron una implementación en una organización, en la cual priorizaron los procesos de soporte a los servicios de TI, entre ellos la gestión de incidentes y la gestión de problemas, así como la gestión de cambios, configuración, entrega y despliegue. En este estudio Lahtela et al. (Lahtela, J“"antti, \& Kaukola, 2010), como resultado de la implementación, rescata la importancia de medir la mejora de los procesos de GSTI, y el impacto que tiene sobre los negocios de la organización.

En la revisión sistemática de la literatura realizada por Melendez et al. (Melendez \& Dávila, 2015), el proceso que la mayoría adopta es el de gestión de incidentes. Según Marrone, Gacenga, Cater-Steel y Kolbe (Marrone, Gacenga, Cater-Steel, \& Kolbe, 2014) normalmente la adopción de modelos de GSTI como ITIL se inicia con el proceso de gestión de incidentes, esto se debe a que su implementación permite reducir el número de interrupciones del servicio y mejora la eficiencia del mismo. Según Melendez et al. (Melendez \& Dávila, 2015) otros de los procesos que suelen implementarse son la gestión de problemas y la gestión de acuerdos de nivel de servicios, debido al interés de las organizaciones por mejorar las actividades de soporte al brindar los servicios de TI (Lahtela, J“"antti, \& Kaukola, 2010).

Como resultado de las implementaciones de modelos de GSTI, las organizaciones han experimentado mejoras en actividades como atención de incidentes, reducción de costos y tiempos, y mejoras en la satisfacción de usuarios (Melendez \& Dávila, 2015). Algunos estudios Küller et al. (Küller, Vogt, Hertweck, \& Grabowski, 2012), Iden y Langeland (Iden \& Langeland, 2010) y Heikkinen y Jantti (Heikkinen \& Jäntti, 2012) coinciden en recomendar ciertos elementos básicos para la adopción de modelos de GSTI, tales como: el desarrollo de capacitaciones sobre modelos de GSTI, procesos de servicios y la identificación de personal con habilidades para la mejora de procesos de servicios. 
Además, según Göbel et al. (Göbel, Cronholm, \& Seigerroth, 2013), Küller et al. (Küller, Vogt, Hertweck, \& Grabowski, 2012), Machado et al. (Machado, Reinehr, \& Malucelli, 2012), Hoerbst et al. (Hoerbst, Hackl, Blomer, \& Ammenwerth, 2011) y Qi (Qi, 2011) la principal dificultad en la adopción de modelos de GSTI es la falta de conocimiento para adoptar modelos y la complejidad que los modelos presentan. Todo lo anterior nos permite afirmar que es necesario el desarrollo de guías o directrices que permitan una fácil adopción de los modelos a la realidad de las organizaciones.

\section{Protocolo de investigación}

Para este trabajo, al ser una investigación exploratoria, se ha optado por usar el estudio de caso cuyo protocolo se ha obtenido a partir de Runeson y Höst (Runeson \& Höst, 2009) y Genero et al. (Genero Bocco, Cruz-Lemus, \& Piattini Velthuis, 2014) . En particular, en el proyecto marco ProCal-ProSer (Dávila, 2013), el proceso seguido se puede apreciar en la Tabla 1 donde las letras P, E y R corresponden a Planificación, Ejecución y Reporte, además presenta un mapeo de esos elementos dentro del artículo.

El objetivo de este estudio (etapa P1-1) es determinar el grado de adopción de las prácticas de gestión de servicios de tecnología de información en pequeñas empresas desarrolladoras de software que ofrecen servicios software. Las prácticas tomadas como referencia son las que están descritas en la ISO/IEC 15504-8 (ISO, 2012)que es un modelo de evaluación para la ISO/IEC 20000-4.

\begin{tabular}{|c|c|c|}
\hline Fase & Etapa & Sección \\
\hline \multirow{6}{*}{ P1: Diseñar el estudio de caso } & (P1-1) Objetivo del estudio & 4.1, introducción \\
\hline & (P1-2) Qué se estudia & $4.2,5.1$ \\
\hline & $(\mathrm{P} 1-3)$ Teoría requerida & Sección 2 y 3 \\
\hline & (P1-4) Preguntas de investigación & $4 \cdot 3$ \\
\hline & (P1-5) Métodos de recolección & 4.4 \\
\hline & (P1-6) Selección de datos & 4.5 \\
\hline $\begin{array}{l}\text { P2: Preparar la recolección de los } \\
\text { datos }\end{array}$ & $\begin{array}{l}(\mathrm{P} 2-1) \text { Definición de cómo se realiza la } \\
\text { recolección }\end{array}$ & 4.6 \\
\hline E1: Recolectar la evidencia & (E1-1) Recolección de los datos & 5.2 \\
\hline \multirow{2}{*}{ E2: Analizar } & (E2-1) Análisis de los datos & $5 \cdot 3$ \\
\hline & (E2-2) Interpretación de los datos & $5 \cdot 4$ \\
\hline R1: Reportar & (R1-1) Realización del informe & Artículo completo \\
\hline
\end{tabular}

Tabla 1 - Fases y etapas del estudio definido en ProCal-Proser (Dávila \& Pessôa, 2015)

Las unidades de que se estudian (etapa P1-2) son pequeñas organizaciones (PO) desarrolladoras de software que ofrecen, además, servicios software, lo que implica un trabajo particular para ellas. Las organizaciones participantes fueron invitadas del grupo de participantes del proyecto ProCal-ProSer. La descripción de las organizaciones y servicios sobre las que se evaluaron las prácticas se presentan en la Sección 5.1. Entonces, el objeto de estudio se basa en tres instancias de análisis y que en adelante 
serán referidas como organizaciones, pues se describirá cada servicio y su contexto de soporte inmediato (unidad organizacional) para no introducir variables que afecten el análisis.

La pregunta de investigación (etapa P1-4) de este estudio es ¿Qué prácticas de la ISO/ IEC 20000 (a través de las correspondientes descritas en la ISO/IEC 15504-8) son consideradas como adoptadas en pequeñas organizaciones desarrolladoras de software que ofrecen servicios?

Los datos a obtener (etapa P1-5) son del tipo cualitativo y se obtuvieron de dos fuentes: una a partir de la evaluación de procesos usando la ISO/IEC 15504 y otra mediante discusiones con miembros del Proyecto sobre los resultados obtenidos.

De la primera fuente, se obtiene información relevante que evidencia el cumplimiento de las prácticas bases de los procesos de la ISO/IEC 20000-4 en las organizaciones participantes, lo que se puede denominar un perfil de prácticas adoptadas. Este perfil fue sometido a miembros del Proyecto para su discusión y consolidación final. El proceso de recolección de datos, para esta primera fuente, se ajustó al proceso descrito en la ISO/ IEC 15504-2 (ISO, 2003) que incluye la planificación de las reuniones con las personas encargadas de los procesos, la revisión de evidencias proporcionadas, la calificación de los procesos y la elaboración del informe de evaluación. Esta recolección se puede considerar como de primer grado (como lo señala Genero et al. (Genero Bocco, CruzLemus, \& Piattini Velthuis, 2014), pues los investigadores estuvimos involucrados de manera directa y en tiempo real en la recogida de datos.

De la segunda fuente, se establecieron discusiones con otros miembros del Proyecto y el equipo evaluador, que según Genero et al. (Genero Bocco, Cruz-Lemus, \& Piattini Velthuis, 2014) pueden ser consideradas como del tipo "No estructurada".

La selección de datos (etapa P1-6) se realizó en dos etapas: en la primera se consideró la información obtenida de los tres procesos de evaluación y en la segunda una discusión entre los investigadores sobre el grupo de prácticas adoptadas en pequeñas organizaciones.

\section{6.(P2-1) Definición de cómo se realiza la recolección}

Para la definición de cómo se realiza la recolección (etapa P2-1) se considera en dos etapa. El primero, es mediante el proceso de evaluación de las tres pequeñas organizaciones, bajo el protocolo de evaluación de la ISO/IEC 15504-2 (ISO, 2003), que tiene definida reglas para realizar la evaluación de los procesos (a través de prácticas y entregables) y verificar su conformidad y el segundo, mediante un proceso de discusión con miembros del Proyecto sobre los resultados obtenidos en la primer etapa, para reafirmar los resultados obtenidos.

\section{Resultados obtenidos}

En esta sección se presenta las instancias o unidades de estudio para efectos de entender aspectos de contexto, luego se presentan los resultados consolidados de las evaluaciones, el análisis de los datos y su interpretación. 


\subsection{Instancias de estudio}

A continuación se describen las organizaciones participantes usando un nombre distinto para cumplir con los acuerdos de confidencialidad.

Caso Lim.Zeta: Lim.Zeta se dedica a prestar servicios tecnológicos e innovadores (incluye tercerización) a diversas empresas a nivel nacional e internacional Para este estudio se procedió a evaluar el servicio que actuaba como intermediador entre dos entidades, que por un lado traduce la información obtenida de una entidad y por otro lado recepciona los datos traducidos, respetando formatos establecidos. Para el servicio ofrecido participan dos empleados de la organización.

Caso Lim.Psi: Lim.Psi presta servicio de soporte técnico de herramientas de software internacionales, actuando como un intermediario entre la entidad extranjera y el cliente, quien hace uso de la herramienta, convirtiéndose Lim.Psi en un socio estratégico para el cliente. De los servicios que presta Lim.Psi, se procedió a evaluar al servicio que consistía en brindar soporte técnico para el uso de la herramienta. Actualmente el servicio es prestado a una empresa de telecomunicaciones nacional, cuyo turno es de 5x8 (5 días por 8 horas). El contrato del servicio se renueva cada cierto tiempo, para realizar las actualizaciones a la herramienta y capacitaciones debido a la rotación del personal del cliente. Para el servicio de soporte técnico participan tres empleados de la organización.

Caso Tru.Omega: Tru.Omega se dedica a prestar soluciones informáticas, enfocadas bajo el concepto de ERP (Enterprise Resource Planning), a diversas empresas clientes, a nivel nacional e internacional de diversos sectores en el negocio, grandes o pequeñas, que requieran mejorar la eficiencia en sus procesos y en la calidad de sus productos o servicios. De los servicios que ofrece esta empresa se procedió a evaluar el servicio de Gestión de Incidencias, el cual consiste de un servicio de soporte técnico para una herramienta ERP creada por ellos mismos. Para brindar el servicio participan cuatro empleados de Tru.Omega.

\section{2. (E1-1) Recolección los datos}

Los datos se recolectaron en dos etapas, uno durante el proceso de evaluación y el otro durante la discusión con los miembros del Proyecto. Para la primera etapa, se recolectan datos del proceso de evaluación realizado a las tres PO, desde donde se obtiene el grado de adopción de cada práctica de la ISO/IEC 20000-4 (que implica evaluar 151 prácticas organizadas en 28 procesos), para ello se utilizó las calificaciones $\mathrm{N}, \mathrm{P}, \mathrm{A}, \mathrm{T}$, basadas en la ISO/IEC 15504-2. Las prácticas base son particulares para cada proceso y la cantidad de prácticas varía según el proceso tal como lo describe la ISO/IEC TS 15504-8:2012 (ISO, 2012).

\section{5•3. (E2-1) Análisis de los datos}

Para obtener el grupo de prácticas adoptadas se procedió a consolidar los resulta-dos del proceso de evaluación. Primero, se determinó en cada organización si la práctica evaluada estaba o no adoptada, usando N, P, A y T (ver Sección 2.2). En las Tablas 2, $3,4,5,6,7$ se muestran los resultados parciales que cada práctica base obtuvo según la organización y el proceso evaluado. Se realizaron consultas a expertos quienes coincidieron en la aprobación de los resultados de cada práctica. 


\section{4. (E2-2) Interpretación de los datos}

En base a los datos de las evaluaciones de cada organización se procedió a consolidar los resultados parciales de cada práctica base según los calificativos N, P, A, T e I. Se introdujo el calificativo I para los casos en que no fuera posible determinar el grado de adopción para el conjunto de respuesta por cada práctica base. Los calificativos se obtuvieron de aplicar las siguientes reglas:

\begin{tabular}{|c|c|c|c|c|c|c|c|c|c|c|c|}
\hline \multirow{2}{*}{ Código } & \multirow{2}{*}{ Proceso } & \multirow{2}{*}{ Caso } & \multicolumn{9}{|c|}{ Prácticas Base } \\
\hline & & & $\mathbf{1}$ & 2 & 3 & 4 & 5 & 6 & 7 & 8 & 9 \\
\hline \multirow{3}{*}{ CON.1 } & \multirow{3}{*}{ Gestión del cambio } & Zeta & $\mathrm{N}$ & $\mathrm{N}$ & $\mathrm{N}$ & $\mathrm{N}$ & $\mathrm{N}$ & $\mathrm{N}$ & $\mathrm{N}$ & & \\
\hline & & Psi & $\mathrm{N}$ & $\mathrm{N}$ & $\mathrm{N}$ & $\mathrm{N}$ & $\mathrm{N}$ & $\mathrm{N}$ & $\mathrm{N}$ & & \\
\hline & & Omega & $\mathrm{N}$ & $\mathrm{N}$ & $\mathrm{N}$ & $\mathrm{N}$ & $\mathrm{N}$ & $\mathrm{N}$ & $\mathrm{N}$ & & \\
\hline \multirow{3}{*}{ CON.2 } & \multirow{3}{*}{ Gestión de la configuración } & Zeta & $\mathrm{N}$ & $\mathrm{N}$ & $\mathrm{P}$ & $\mathrm{P}$ & $\mathrm{P}$ & & & & \\
\hline & & Psi & $\mathrm{T}$ & $\mathrm{N}$ & A & $\mathrm{P}$ & $\mathrm{P}$ & & & & \\
\hline & & Omega & $\mathrm{T}$ & $\mathrm{N}$ & A & $\mathrm{N}$ & $\mathrm{N}$ & & & & \\
\hline \multirow{3}{*}{ CON.3 } & \multirow{3}{*}{ Gestión del release y despliegue } & Zeta & $\mathrm{A}$ & $\mathrm{P}$ & $\mathrm{P}$ & A & $\mathrm{T}$ & $\mathrm{P}$ & $\mathrm{N}$ & A & \\
\hline & & Psi & $\mathrm{T}$ & A & $\mathrm{N}$ & $\mathrm{T}$ & $\mathrm{T}$ & $\mathrm{T}$ & $\mathrm{N}$ & $\mathrm{T}$ & \\
\hline & & Omega & A & A & $\mathrm{A}$ & $\mathrm{N}$ & $\mathrm{T}$ & $\mathrm{T}$ & $\mathrm{T}$ & $\mathrm{T}$ & \\
\hline
\end{tabular}

Tabla 2 - Evaluación de las prácticas base para los procesos de control

- Calificativo T: si al menos dos PO obtuvieron, para la práctica analizada, calificación T y en la otra A o T. Por ejemplo si las calificaciones obtenidas son T, T y A entonces para esta práctica se obtiene el calificativo final de T.

- Calificativo A: si al menos dos PO obtuvieron, para la práctica analizada, calificación A y en la otra A o T. Por ejemplo si las calificaciones obtenidas son A, A y T entonces para esta práctica se obtiene el calificativo final de A.

- Calificativo P: si al menos dos PO obtuvieron calificación P y en la otra N. Por ejemplo si las calificaciones obtenidas son $\mathrm{P}, \mathrm{P}$ y N entonces para esta práctica se obtiene el calificativo final de $\mathrm{P}$.

- Calificativo N: si al menos dos PO obtuvieron calificación N y en el resto cualquier otra calificación entonces para esta práctica se obtiene el calificativo final de $\mathrm{N}$.

- Calificativo I: se obtiene para los casos que no se han mencionado en los anteriores calificativos.

De lo cual se puede resumir a nivel de proceso los resultados finales de las 151 prácticas base que consta en la ISO/IEC 20000-4, como se aprecia en la Tabla 8.

A partir de la calificación anterior se procedió a determinar las prácticas que se encuentran adoptadas, indeterminadas o no adoptadas. Para ello se agrupó de la siguiente manera: (i) Adoptada: agrupación de aquellas que son A o T; (ii) Indeterminado: aquellas que son I; y (iii) No adoptado: agrupación de aquellas que son $\mathrm{N}$ o P. Siendo los resultados a nivel de proceso los siguientes: 


\begin{tabular}{|c|c|c|c|c|c|c|c|c|c|c|c|}
\hline \multirow{2}{*}{ Código } & \multirow{2}{*}{ Proceso } & \multirow{2}{*}{ Caso } & \multicolumn{9}{|c|}{ Prácticas Base } \\
\hline & & & $\mathbf{1}$ & 2 & 3 & 4 & 5 & 6 & 7 & 8 & 9 \\
\hline \multirow{3}{*}{ SMS.1 } & \multirow{3}{*}{ Auditoría } & Zeta & $\mathrm{P}$ & $\mathrm{P}$ & $\mathrm{P}$ & $\mathrm{N}$ & $\mathrm{P}$ & $\mathrm{N}$ & & & \\
\hline & & Psi & $\mathrm{P}$ & $\mathrm{N}$ & $\mathrm{P}$ & $\mathrm{P}$ & A & A & & & \\
\hline & & Omega & $\mathrm{P}$ & $\mathrm{N}$ & $\mathrm{N}$ & $\mathrm{N}$ & $\mathrm{N}$ & $\mathrm{N}$ & & & \\
\hline \multirow{3}{*}{ SMS.2 } & \multirow{3}{*}{ Mejora } & Zeta & $\mathrm{P}$ & $\mathrm{P}$ & $\mathrm{N}$ & $\mathrm{N}$ & $\mathrm{N}$ & & & & \\
\hline & & Psi & $\mathrm{A}$ & $\mathrm{P}$ & $\mathrm{N}$ & $\mathrm{N}$ & $\mathrm{N}$ & & & & \\
\hline & & Omega & $\mathrm{A}$ & A & $\mathrm{A}$ & $\mathrm{T}$ & $\mathrm{T}$ & & & & \\
\hline \multirow{3}{*}{ SMS.3 } & \multirow{3}{*}{ Gestión del elemento de información } & Zeta & $\mathrm{A}$ & A & $\mathrm{T}$ & $\mathrm{T}$ & $\mathrm{T}$ & & & & \\
\hline & & Psi & $\mathrm{A}$ & A & A & A & $\mathrm{T}$ & & & & \\
\hline & & Omega & $\mathrm{T}$ & A & A & $\mathrm{T}$ & $\mathrm{T}$ & & & & \\
\hline \multirow{3}{*}{ SMS.4 } & \multirow{3}{*}{ Revisión de la alta dirección } & Zeta & A & A & A & A & A & & & & \\
\hline & & Psi & $\mathrm{P}$ & $\mathrm{N}$ & $\mathrm{P}$ & A & $\mathrm{N}$ & & & & \\
\hline & & Omega & $\mathrm{A}$ & $\mathrm{A}$ & $\mathrm{N}$ & $\mathrm{T}$ & $\mathrm{T}$ & & & & \\
\hline \multirow{3}{*}{ SMS.5 } & \multirow{3}{*}{ Gestión de recursos } & Zeta & $\mathrm{T}$ & $\mathrm{T}$ & $\mathrm{T}$ & $\mathrm{P}$ & & & & & \\
\hline & & Psi & $\mathrm{T}$ & $\mathrm{T}$ & $\mathrm{T}$ & $\mathrm{T}$ & & & & & \\
\hline & & Omega & $\mathrm{T}$ & $\mathrm{T}$ & $\mathrm{T}$ & $\mathrm{T}$ & & & & & \\
\hline \multirow{3}{*}{ SMS.6 } & \multirow{3}{*}{ Gestión del riesgo } & Zeta & $\mathrm{N}$ & $\mathrm{N}$ & $\mathrm{N}$ & $\mathrm{N}$ & $\mathrm{P}$ & & & & \\
\hline & & Psi & $\mathrm{P}$ & $\mathrm{N}$ & $\mathrm{P}$ & $\mathrm{N}$ & $\mathrm{N}$ & & & & \\
\hline & & Omega & $\mathrm{A}$ & $\mathrm{N}$ & $\mathrm{A}$ & $\mathrm{N}$ & $\mathrm{P}$ & & & & \\
\hline \multirow{3}{*}{ SMS.7 } & \multirow{3}{*}{ Medición del servicio } & Zeta & $\mathrm{N}$ & $\mathrm{N}$ & $\mathrm{N}$ & $\mathrm{N}$ & $\mathrm{N}$ & & & & \\
\hline & & Psi & $\mathrm{N}$ & $\mathrm{N}$ & $\mathrm{N}$ & $\mathrm{N}$ & $\mathrm{N}$ & & & & \\
\hline & & Omega & A & A & A & A & $\mathrm{T}$ & & & & \\
\hline \multirow{3}{*}{ SMS.8 } & \multirow{3}{*}{ Establecimiento y mejora del SMS } & Zeta & $\mathrm{P}$ & $\mathrm{P}$ & $\mathrm{N}$ & $\mathrm{N}$ & & & & & \\
\hline & & Psi & $\mathrm{P}$ & $\mathrm{N}$ & $\mathrm{N}$ & $\mathrm{A}$ & & & & & \\
\hline & & Omega & $\mathrm{P}$ & $\mathrm{P}$ & $\mathrm{N}$ & A & & & & & \\
\hline \multirow{3}{*}{ SMS.9 } & \multirow{3}{*}{ Implementación y operación del SMS } & Zeta & $\mathrm{N}$ & $\mathrm{N}$ & $\mathrm{N}$ & $\mathrm{N}$ & $\mathrm{N}$ & & & & \\
\hline & & Psi & $\mathrm{A}$ & A & $\mathrm{A}$ & $\mathrm{A}$ & $\mathrm{P}$ & & & & \\
\hline & & Omega & $\mathrm{A}$ & $\mathrm{A}$ & $\mathrm{A}$ & A & A & & & & \\
\hline
\end{tabular}

Tabla 3 - Evaluación de las prácticas para los procesos del sistema de gestión de servicios 


\begin{tabular}{|c|c|c|c|c|c|c|c|c|c|c|c|}
\hline \multirow{2}{*}{ Código } & \multirow{2}{*}{ Proceso } & \multirow{2}{*}{ Caso } & \multicolumn{9}{|c|}{ Prácticas Base } \\
\hline & & & $\mathbf{1}$ & 2 & 3 & 4 & 5 & 6 & 7 & 8 & 9 \\
\hline \multirow{3}{*}{ RES. 1} & \multirow{3}{*}{ Gestión de incidentes } & Zeta & A & A & $\mathrm{T}$ & $\mathrm{P}$ & $\mathrm{T}$ & & & & \\
\hline & & Psi & $\mathrm{A}$ & A & A & $\mathrm{T}$ & $\mathrm{T}$ & & & & \\
\hline & & Omega & $\mathrm{T}$ & $\mathrm{T}$ & $\mathrm{T}$ & $\mathrm{T}$ & $\mathrm{A}$ & & & & \\
\hline \multirow{3}{*}{ RES.2 } & \multirow{3}{*}{ Gestión de solicitud de servicio } & Zeta & $\mathrm{A}$ & A & $\mathrm{T}$ & $\mathrm{P}$ & $\mathrm{T}$ & & & & \\
\hline & & Psi & $\mathrm{A}$ & A & A & $\mathrm{T}$ & $\mathrm{T}$ & & & & \\
\hline & & Omega & $\mathrm{T}$ & & $\mathrm{T}$ & $\mathrm{T}$ & A & & & & \\
\hline \multirow{3}{*}{ RES.3 } & \multirow{3}{*}{ Gestión del problema } & Zeta & $\mathrm{A}$ & A & $\mathrm{T}$ & $\mathrm{P}$ & $\mathrm{A}$ & $\mathrm{T}$ & & & \\
\hline & & Psi & $\mathrm{P}$ & $\mathrm{N}$ & $\mathrm{T}$ & $\mathrm{T}$ & $\mathrm{T}$ & $\mathrm{T}$ & & & \\
\hline & & Omega & A & $\mathrm{N}$ & A & A & $\mathrm{N}$ & A & & & \\
\hline
\end{tabular}

Tabla 4 - Evaluación de las prácticas base para los procesos de resolución

\begin{tabular}{|c|c|c|c|c|c|c|c|c|c|c|c|}
\hline \multirow{2}{*}{ Código } & \multirow{2}{*}{ Proceso } & \multirow{2}{*}{ Caso } & \multicolumn{9}{|c|}{ Prácticas Base } \\
\hline & & & $\mathbf{1}$ & 2 & 3 & 4 & 5 & 6 & 7 & 8 & 9 \\
\hline \multirow{3}{*}{ REL.1 } & \multirow{3}{*}{ Gestión de las relaciones del negocio } & Zeta & A & A & A & A & $\mathrm{N}$ & $\mathrm{N}$ & A & $\mathrm{N}$ & $\mathrm{N}$ \\
\hline & & Psi & $\mathrm{A}$ & $\mathrm{N}$ & $\mathrm{T}$ & $\mathrm{T}$ & $\mathrm{N}$ & $\mathrm{N}$ & $\mathrm{N}$ & $\mathrm{N}$ & $\mathrm{N}$ \\
\hline & & Omega & $\mathrm{T}$ & A & A & A & $\mathrm{N}$ & $\mathrm{P}$ & A & $\mathrm{N}$ & $\mathrm{N}$ \\
\hline \multirow{3}{*}{ REL.2 } & \multirow{3}{*}{ Gestión de suministradores } & Zeta & $\mathrm{N}$ & $\mathrm{N}$ & $\mathrm{N}$ & $\mathrm{N}$ & $\mathrm{N}$ & $\mathrm{N}$ & & & \\
\hline & & Psi & $\mathrm{T}$ & $\mathrm{T}$ & $\mathrm{T}$ & $\mathrm{T}$ & A & A & & & \\
\hline & & Omega & A & $\mathrm{T}$ & $\mathrm{T}$ & $\mathrm{T}$ & $\mathrm{P}$ & $\mathrm{N}$ & & & \\
\hline
\end{tabular}

Tabla 5 - Evaluación de las prácticas base para los procesos de relaciones

- Los procesos de control (CON), que agrupa a Gestión del cambio (CON.1), Gestión de la configuración (CON.2) y el proceso de Gestión del release y despliegue (CON.3), tienen en conjunto 20 prácticas de las cuales se puede señalar que $15 \%$ de ellas se consideran adoptadas, 50\% de ellas no adoptadas, y sobre el resto (35\%) no es posible determinar su adopción.

- Los procesos de resolución (RES), que agrupa Gestión de incidentes (RES.1), Gestión de solicitud de servicio (RES.2) y Gestión del problema (RES.3), tienen 16 prácticas en conjunto de las cuales se puede señalar que 63.5\% de las prácticas son adoptadas, no se cuentan con prácticas no adoptadas y 38.5\% no es posible determinar la adopción. 


\begin{tabular}{|c|c|c|c|c|c|c|c|c|c|c|c|}
\hline \multirow{2}{*}{ Código } & \multirow{2}{*}{ Proceso } & \multirow{2}{*}{ Caso } & \multicolumn{9}{|c|}{ Prácticas Base } \\
\hline & & & $\mathbf{1}$ & 2 & 3 & 4 & 5 & 6 & 7 & 8 & 9 \\
\hline \multirow{3}{*}{ DTR.1 } & \multirow{3}{*}{ Requerimientos del servicio } & Zeta & A & $\mathrm{T}$ & $\mathrm{T}$ & $\mathrm{T}$ & $\mathrm{N}$ & & & & \\
\hline & & Psi & $\mathrm{T}$ & $\mathrm{T}$ & $\mathrm{T}$ & $\mathrm{T}$ & $\mathrm{T}$ & & & & \\
\hline & & Omega & A & $\mathrm{A}$ & $\mathrm{T}$ & $\mathrm{N}$ & $\mathrm{N}$ & & & & \\
\hline \multirow{3}{*}{ DTR.2 } & \multirow{3}{*}{ Diseño del servicio } & Zeta & $\mathrm{T}$ & $\mathrm{A}$ & A & $\mathrm{T}$ & & & & & \\
\hline & & Psi & $\mathrm{T}$ & $\mathrm{T}$ & $\mathrm{T}$ & $\mathrm{T}$ & & & & & \\
\hline & & Omega & A & $\mathrm{A}$ & A & A & & & & & \\
\hline \multirow{3}{*}{ DTR.3 } & \multirow{3}{*}{ Transición del servicio } & Zeta & $\mathrm{T}$ & $\mathrm{A}$ & A & A & & & & & \\
\hline & & Psi & $\mathrm{T}$ & $\mathrm{A}$ & $\mathrm{T}$ & $\mathrm{T}$ & & & & & \\
\hline & & Omega & $\mathrm{T}$ & $\mathrm{A}$ & A & $\mathrm{T}$ & & & & & \\
\hline \multirow{3}{*}{ DTR.4 } & \multirow{3}{*}{ Planeamiento del servicio } & Zeta & A & $\mathrm{N}$ & $\mathrm{A}$ & $\mathrm{N}$ & $\mathrm{N}$ & & & & \\
\hline & & Psi & A & $\mathrm{A}$ & $\mathrm{T}$ & A & $\mathrm{A}$ & & & & \\
\hline & & Omega & $\mathrm{T}$ & $\mathrm{N}$ & $\mathrm{T}$ & A & $\mathrm{N}$ & & & & \\
\hline
\end{tabular}

Tabla 6. - Evaluación de las prácticas base para los procesos de diseño y transición de servicios nuevos o modificados

- Los procesos generales del sistema de gestión de servicios (SMS), que agrupa Auditoría (SMS.1), Mejora (SMS.2), Gestión del elemente de información (SMS.3), Revisión de la alta dirección (SMS.4), Gestión de recursos (SMS.5), Gestión del riesgo (SMS.6), Medición del servicio (SMS.7), Establecimiento y mejora del SMS (SMS.8), y el proceso de Implementación y operación del SMS (SMS.9), tienen 44 prácticas en conjunto, entre las cuales se determinó 20\% de prácticas adoptadas, 23\% de prácticas no adoptadas y 57\% de prácticas en las que no es posible determinar la adopción.

- Los procesos de relaciones (REL) que agrupa Gestión de las relaciones de negocio (REL.1) y Gestión de suministradores (REL.2), tienen 15 prácticas de las cuales se encontró, 20\% de ellas como adoptadas, 27\% no adoptadas y 53\% de prácticas que no se pudo determinar su adopción.

- Los procesos de diseño y transición de servicios nuevos o modificados (DTR), los cuales son: Requerimientos del servicio (DTR.1), Diseño del servicio (DTR.2), Transición del servicio (DTR.3) y Planeamiento del servicio (DTR.4); tienen en conjunto 18 prácticas de las cuales se puede señalar que $72 \%$ de ellas son adoptadas, o\% prácticas no adoptadas, y 28\% de prácticas no es posible determinar la adopción.

- Los procesos de entrega del servicio (SDE), que agrupa Presupuesto y contabilidad de los servicios TI (SDE.1), Gestión de la capacidad (SDE.2), 
Gestión de seguridad de información (SDE.3), Gestión de disponibilidad del servicio (SDE.4), Gestión de la continuidad del servicio (SDE.5), Gestión del nivel de servicio (SDE.6) y Reporte del servicio (SDE.7), tienen 38 prácticas de los cuales se obtuvo que $24 \%$ son prácticas adoptadas, $26 \%$ no son adoptadas y $50 \%$ no es posible determinar la adopción.

\begin{tabular}{|c|c|c|c|c|c|c|c|c|c|c|c|}
\hline \multirow{2}{*}{ Código } & \multirow{2}{*}{ Proceso } & \multirow{2}{*}{ Caso } & \multicolumn{9}{|c|}{ Prácticas Base } \\
\hline & & & 1 & 2 & 3 & 4 & 5 & 6 & 7 & 8 & 9 \\
\hline \multirow{3}{*}{ SDE.1 } & \multirow{3}{*}{$\begin{array}{l}\text { Presupuesto y contabilidad de los } \\
\text { servicios TI }\end{array}$} & Zeta & $\mathrm{N}$ & $\mathrm{N}$ & $\mathrm{N}$ & $\mathrm{P}$ & A & & & & \\
\hline & & Psi & $\mathrm{T}$ & $\mathrm{T}$ & $\mathrm{N}$ & $\mathrm{P}$ & $\mathrm{T}$ & & & & \\
\hline & & Omega & $\mathrm{T}$ & $\mathrm{A}$ & A & A & $\mathrm{T}$ & & & & \\
\hline \multirow{3}{*}{ SDE.2 } & \multirow{3}{*}{ Gestión de la capacidad } & Zeta & $\mathrm{A}$ & $\mathrm{P}$ & $\mathrm{N}$ & $\mathrm{N}$ & & & & & \\
\hline & & Psi & $\mathrm{T}$ & A & $\mathrm{T}$ & $\mathrm{T}$ & & & & & \\
\hline & & Omega & $\mathrm{T}$ & $\mathrm{T}$ & A & $\mathrm{T}$ & & & & & \\
\hline \multirow{3}{*}{ SDE.3 } & \multirow{3}{*}{ Gestión de seguridad de información } & Zeta & $\mathrm{P}$ & $\mathrm{N}$ & $\mathrm{N}$ & $\mathrm{N}$ & $\mathrm{P}$ & $\mathrm{N}$ & $\mathrm{N}$ & $\mathrm{N}$ & $\mathrm{N}$ \\
\hline & & Psi & $\mathrm{T}$ & $\mathrm{P}$ & $\mathrm{P}$ & $\mathrm{N}$ & $\mathrm{N}$ & $\mathrm{N}$ & $\mathrm{N}$ & A & $\mathrm{N}$ \\
\hline & & Omega & A & $\mathrm{N}$ & $\mathrm{N}$ & $\mathrm{N}$ & $\mathrm{N}$ & $\mathrm{N}$ & A & $\mathrm{T}$ & $\mathrm{N}$ \\
\hline \multirow{3}{*}{ SDE.4 } & \multirow{3}{*}{ Gestión de disponibilidad del servicio } & Zeta & A & $\mathrm{N}$ & A & $\mathrm{T}$ & $\mathrm{A}$ & A & $\mathrm{N}$ & & \\
\hline & & Psi & $\mathrm{T}$ & A & $\mathrm{T}$ & $\mathrm{T}$ & $\mathrm{N}$ & $\mathrm{N}$ & $\mathrm{N}$ & & \\
\hline & & Omega & $\mathrm{T}$ & $\mathrm{A}$ & $\mathrm{P}$ & $\mathrm{T}$ & $\mathrm{N}$ & $\mathrm{N}$ & $\mathrm{N}$ & & \\
\hline \multirow{3}{*}{ SDE.5 } & \multirow{3}{*}{ Gestión de la continuidad del servicio } & Zeta & $\mathrm{A}$ & $\mathrm{P}$ & A & $\mathrm{N}$ & & & & & \\
\hline & & Psi & $\mathrm{T}$ & A & $\mathrm{T}$ & $\mathrm{N}$ & & & & & \\
\hline & & Omega & $\mathrm{T}$ & $\mathrm{A}$ & $\mathrm{P}$ & $\mathrm{N}$ & & & & & \\
\hline \multirow{3}{*}{ SDE.6 } & \multirow{3}{*}{ Gestión del nivel de servicio } & Zeta & $\mathrm{A}$ & $\mathrm{T}$ & $\mathrm{N}$ & A & $\mathrm{N}$ & & & & \\
\hline & & Psi & $\mathrm{N}$ & $\mathrm{N}$ & $\mathrm{N}$ & $\mathrm{N}$ & $\mathrm{N}$ & & & & \\
\hline & & Omega & A & $\mathrm{T}$ & $\mathrm{N}$ & $\mathrm{A}$ & $\mathrm{N}$ & & & & \\
\hline \multirow{3}{*}{ SDE.7 } & \multirow{3}{*}{ Reporte del servicio } & Zeta & $\mathrm{T}$ & $\mathrm{T}$ & $\mathrm{T}$ & $\mathrm{T}$ & & & & & \\
\hline & & Psi & $\mathrm{T}$ & $\mathrm{T}$ & $\mathrm{T}$ & $\mathrm{T}$ & & & & & \\
\hline & & Omega & $\mathrm{T}$ & $\mathrm{T}$ & $\mathrm{T}$ & $\mathrm{T}$ & & & & & \\
\hline
\end{tabular}

Tabla 7 - Evaluación de las prácticas base para los procesos de entrega del servicio 


\begin{tabular}{|c|c|c|c|c|c|c|c|c|c|c|}
\hline \multirow{2}{*}{ Código } & \multirow{2}{*}{ Proceso } & \multicolumn{9}{|c|}{ Prácticas Base } \\
\hline & & $\mathbf{1}$ & 2 & 3 & 4 & 5 & 6 & 7 & 8 & 9 \\
\hline CON.1 & Gestión del cambio & $\mathrm{N}$ & $\mathrm{N}$ & $\mathrm{N}$ & $\mathrm{N}$ & $\mathrm{N}$ & $\mathrm{N}$ & $\mathrm{N}$ & & \\
\hline CON.2 & Gestión de la configuración & $\mathrm{I}$ & $\mathrm{N}$ & I & $\mathrm{P}$ & $\mathrm{P}$ & & & & \\
\hline CON.3 & Gestión del release y despliegue & $\mathrm{A}$ & I & I & I & $\mathrm{F}$ & I & I & $\mathrm{F}$ & \\
\hline SMS.1 & Auditoría & $\mathrm{P}$ & $\mathrm{N}$ & $\mathrm{P}$ & $\mathrm{N}$ & I & I & & & \\
\hline SMS.2 & Mejora & I & I & I & I & $\mathrm{I}$ & & & & \\
\hline SMS.3 & Gestión del elemento de información & $\mathrm{A}$ & $\mathrm{A}$ & $\mathrm{A}$ & $\mathrm{F}$ & $\mathrm{F}$ & & & & \\
\hline SMS.4 & Revisión de la alta dirección & $\mathrm{I}$ & I & I & $\mathrm{A}$ & $\mathrm{I}$ & & & & \\
\hline SMS.5 & Gestión de recursos & $\mathrm{F}$ & $\mathrm{F}$ & $\mathrm{F}$ & I & & & & & \\
\hline SMS.6 & Gestión del riesgo & I & $\mathrm{N}$ & I & $\mathrm{N}$ & $\mathrm{P}$ & & & & \\
\hline SMS.7 & Medición del servicio & I & I & $\mathrm{I}$ & I & $\mathrm{I}$ & & & & \\
\hline SMS.8 & SGS Establecimiento y mejora & $\mathrm{P}$ & $\mathrm{P}$ & $\mathrm{N}$ & I & & & & & \\
\hline SMS.9 & Implementación y operación del SGS & I & $\mathrm{I}$ & I & I & $\mathrm{I}$ & & & & \\
\hline RES.1 & Gestión de incidentes & A & $\mathrm{A}$ & $\mathrm{F}$ & $\mathrm{I}$ & $\mathrm{F}$ & & & & \\
\hline RES.2 & Gestión de solicitud de servicio & $\mathrm{A}$ & A & $\mathrm{F}$ & I & $\mathrm{F}$ & & & & \\
\hline RES.3 & Gestión del problema & $\mathrm{I}$ & $\mathrm{I}$ & $\mathrm{F}$ & $\mathrm{I}$ & $\mathrm{I}$ & $\mathrm{F}$ & & & \\
\hline REL.1 & Gestión de las relaciones del negocio & A & $\mathrm{I}$ & $\mathrm{A}$ & A & $\mathrm{N}$ & $\mathrm{N}$ & I & $\mathrm{N}$ & $\mathrm{N}$ \\
\hline REL.2 & Gestión de suministradores & I & I & $\mathrm{I}$ & I & I & $\mathrm{I}$ & & & \\
\hline DTR.1 & Requerimientos del servicio & A & $\mathrm{F}$ & $\mathrm{F}$ & $\mathrm{I}$ & $\mathrm{I}$ & & & & \\
\hline DTR.2 & Diseño del servicio & $\mathrm{F}$ & A & $\mathrm{A}$ & $\mathrm{F}$ & & & & & \\
\hline DTR.3 & Transición del servicio & $\mathrm{F}$ & A & $\mathrm{A}$ & $\mathrm{F}$ & & & & & \\
\hline DTR.4 & Planeamiento del servicio & $\mathrm{A}$ & $\mathrm{I}$ & $\mathrm{F}$ & $\mathrm{I}$ & $\mathrm{I}$ & & & & \\
\hline SDE.1 & Presupuesto y contabilidad de los servicios TI & I & $\mathrm{I}$ & I & I & $\mathrm{F}$ & & & & \\
\hline SDE.2 & Gestión de la capacidad & $\mathrm{F}$ & I & I & $\mathrm{I}$ & & & & & \\
\hline SDE.3 & Gestión de seguridad de información & I & $\mathrm{N}$ & $\mathrm{N}$ & $\mathrm{N}$ & $\mathrm{N}$ & $\mathrm{N}$ & I & $\mathrm{I}$ & $\mathrm{N}$ \\
\hline SDE.4 & Gestión de disponibilidad del servicio & $\mathrm{F}$ & $\mathrm{I}$ & I & $\mathrm{F}$ & $\mathrm{I}$ & I & $\mathrm{N}$ & & \\
\hline SDE.5 & Gestión de la continuidad del servicio & $\mathrm{F}$ & $\mathrm{I}$ & I & $\mathrm{N}$ & & & & & \\
\hline SDE.6 & Gestión del nivel de servicio & I & I & $\mathrm{N}$ & $\mathrm{I}$ & $\mathrm{N}$ & & & & \\
\hline SDE.7 & Reporte del servicio & $\mathrm{F}$ & $\mathrm{F}$ & $\mathrm{F}$ & $\mathrm{F}$ & & & & & \\
\hline
\end{tabular}

Tabla 8 - Calificación para cada práctica base de la ISO/IEC 20000-4 
Consolidando todo lo anterior, se obtiene que de las 151 prácticas de la ISO/IEC 20000 (evaluado a través de las prácticas base de la ISO/IEC 20000-4 y la ISO/IEC 155048) se puede señalar que 47 prácticas son adoptadas (31\%), 34 prácticas (23\%) no son adoptadas y no se pudo determinar sobre 70 prácticas (46\%) las cuales han sido referidas como indeterminadas anteriormente.

\section{Discusión final y trabajo futuro}

Considerando que el trabajo presenta una investigación exploratoria sobre la base de un estudio de caso en tres empresas, se tiene que los resultados muestran hallazgos que nos permitirán establecer una investigación en mayor profundidad.

En la evaluación y discusión de los resultados obtenidos, se logró identificar que el grupo prácticas que han sido adoptadas por las PO, en su mayoría están relaciona-das a los procesos de RES con $63 \%$ y los de DTR con $72 \%$.

Las prácticas del proceso RES que tienen un 63\% de adopción son de gestión de incidentes (GI) y solicitud de servicio (SS). Las prácticas de GI, al ser parte de las operaciones de los servicios que brindan las PO son las más importantes para ellas, por lo tanto, más usadas. Este resultado refuerza algunas investigaciones como las de Liu et al. (Liu, Gao, Luo, \& Wan, 2011), Lahtela et al. (Lahtela, J“antti, \& Kaukola, 2010)y Marrone et al. (Marrone, Gacenga, Cater-Steel, \& Kolbe, 2014), en las cuales se mencionan que las organizaciones inician la adopción priorizando la gestión de incidentes como soporte a sus operaciones diarias.

Las prácticas de DTR que fueron adoptadas son principalmente sobre requisitos del servicio, diseño y transición del servicio, es decir, las PO se preocupan por el establecimiento de los servicios que ofrecen a sus clientes. Esto refuerza lo señalado por Lahtela et al. (Lahtela, J“antti, \& Kaukola, 2010) que menciona las actividades de entrega y despliegue como uno de las implementadas en las organizaciones que ofrecen servicios. Sin embargo, cabe señalar que las prácticas referidas a gestión de problemas en su mayoría son indeterminadas. Si bien, durante las entrevistas las organizaciones indicaban que resolvían los problemas, la forma en que lo hacían (de manera desordenada y reactiva) no reflejaba el cumplimiento de las prácticas evaluadas de la ISO/IEC 155048. Esta situación amerita una investigación futura y de mayor profundidad sobre estas prácticas.

Entre las prácticas no adoptadas están las referidas a las actividades de control como gestión del cambio y configuración, que aparenta ser de menor interés.

Además en la entrevista a los participantes de las organizaciones se observó que para el desarrollo del servicio, se guían más de su experiencia vivida en situaciones similares que en aplicar prácticas recogidas en modelos, tal como la ISO/IEC 20000 o ITIL. Esta falta de adopción de prácticas genera un espacio de investigación para identificar las causas o factores que influyen en su adopción, las que posteriormente deben ser consideradas en los planes de mejora de procesos.

Como trabajo futuro se realizará un análisis de las prácticas adoptadas respecto del modelo PCPS para servicios y que actualmente se encuentra en la fase de pruebas controladas en 
empresas. Asimismo, se extenderá el estudio a un grupo mayor de organizaciones para confirmar los resultados obtenidos. La ampliación cubrirá dos grupos de organizaciones, una de desarrolladoras de software y otras que no desarrollan software evaluando en ambos casos la ISO/IEC 20000-4 y el modelo desarrollado por nuestro Proyecto.

\section{Agradecimientos}

Este trabajo ha sido realizado dentro del proyecto ProCal-ProSer financiado por Innóvate Perú bajo el Contrato 210-FINCYT-IA-2013 y parcialmente soportado por el Departamento de Ingeniería y el Grupo de Investigación y Desarrollo de Ingeniería de Software (GIDIS) de la Pontificia Universidad Católica del Perú.

\section{Referencias}

Arcilla, M., Calvo-Manzano, J., \& San Feliu, T. (2013). Building an IT service catalog in a small company as the main input for the IT financial management. Journal Computer Standards \& Interfaces, 36(1), 42-53.

Dávila, A. (o1 de Diciembre de 2013). Presentación. ProCal-ProSer. Recuperado el 14 de Marzo de 2015, de sitio web de ProCal-ProSer: https://sites.google.com/a/pucp. pe/procal-proser/

Dávila, A., \& Pessôa, M. (2015). Factores en la adopción de prácticas de la ISO/IEC 29110: un estudio de caso de una micro empresa desarrolladora de software. Simposio Latinoamericano de Ingeniería de Software. En XLI Conferencia Latinoamericana en Informática (págs. 1-7). Arequipa: CLEI2015.

Dávila, A., Basurto, C., Flores, L., Manrique, R., Arisaca, R., Sánchez, J., \& Pessôa, M. (2012). The peruvian component of Competisoft project: Lesson learned from academic perspective. 38th Latin America Conference on Informatics CLEI 2012 Conference Proceedings (págs. 1-7). Medellin: IEEE.

Genero Bocco, M. F., Cruz-Lemus, J. A., \& Piattini Velthuis, M. G. (2014). Métodos de Investigación en Ingeniería de Software (Primera ed.). Madrid, España: RA-MA.

Göbel, H., Cronholm, S., \& Seigerroth, U. (2013). Towards an agile method for ITSM self-assessment: A Design Science Research Approach. International Conference on Management, Leadership \& Governance, (págs. 135-142).

Heikkinen, S., \& Jäntti, M. (2012). Identifying IT Service Management Challenges: A Case Study in Two IT Service Provider Companies. 23rd International Workshop on Database and Expert Systems Applications (págs. 55-59). Viena: IEEE.

Hoerbst, A., Hackl, W., Blomer, R., \& Ammenwerth, E. (2011). The status of IT service

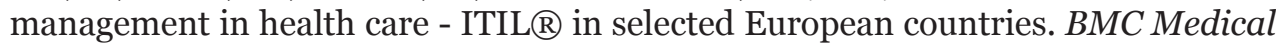
Informatics and Decision Making(11), 1-12.

Iden, J., \& Langeland, L. (2010). Setting the Stage for a Successful ITIL Adoption: A Delphi Study of IT Experts in the Norwegian Armed Forces. Information Systems Management, 27(2), 103-112. 
ISO. (2003). ISO/IEC 15504-2: 2003 Information technology -- Process assessment -- Part 2: Performing an assessment. Geneva: ISO.

ISO. (2010). ISO/IECTR 200oo-4:2010 Information technology--Service management -- Part 4: Process reference model. Geneva: ISO.

ISO. (2011). ISO/IEC 200oo-1:2011 Information technology -- Service management -- Part 1: Service management system requirements. Geneva: ISO.

ISO. (2012). ISO/IEC TS 15504-8:2012 Information technology -- Process assessment -- Part 8: An exemplar process assessment model for IT service management. Geneva: ISO.

ITIL. (2015). Information Technology Infrastructure Library. Recuperado el 2015, de Open Guide: http://www.itlibrary.org/

Küller, P., Vogt, M., Hertweck, D., \& Grabowski, M. (2012). IT Service Management for Small and Medium-Sized Enterprises: A Domain Specific Approach. Journal of Innovation Management in Small \& Medium Enterprises, 2012, 1-17.

Lahtela, A., J“antti, M., \& Kaukola, J. (2010). Implementing an ITIL-Based IT Service Management Measurement System. ICDS '1o Fourth International Conference on Digital Society, (págs. 249-254).

Liu, M., Gao, Z., Luo, W., \& Wan, J. (2011). Case study on IT service management process evaluation framework based on ITIL. 2011 International Conference on Business Management and Electronic Information (págs. 199-202). Guangzhou: IEEE.

Machado, R. F., Reinehr, S., \& Malucelli, A. (2012). Towards a maturity model for IT service management applied to small and medium enterprises. 19th European Conference on Systems, Software and Services Process Improvement, (págs. 157168). Viena.

Marrone, M., Gacenga, F., Cater-Steel, A., \& Kolbe, L. (2014). IT Service Management: A Cross-national Study of ITIL Adoption. Communications of the Association for Information Systems, 34(49), 865-892.

Mejia, J., Muñoz, M., \& Rocha, Á. (2014). La Mejora de Procesos de Software a través de la. E1(DOI: 10.4304/risti.e1.vii-xi), VII,VIII,IX,X,XI.

Melendez, K., \& Dávila, A. (2015). Information Technology Services Management Models Applied to Medium and Small Organizations: A Systematic Literature Review. Computer Standards \& Interfaces, 47, 120-127.

Mesquida, A. L., Mas, A., San, T., \& Arcilla, M. (2014). Integración de Estándares de Gestión de TI mediante MIN-ITs. Revista Ibérica de Sistemas e Tecnologias de Informação, E1(DOI: 10.4304/risti.e1.31-45), 31-45.

Qi, L. (2011). Operation maintenance and management model on informationization system of small and medium enterprises. 2011 2nd International Conference on Artificial Intelligence, Management Science and Electronic Commerce (págs. 6700-6703). Deng Leng: IEEE. 
Rocha, Á., \& Freixo, J. (2015). Information Architecture for Quality Management Support in Hospitals. Journal of Medical Systems, 39(10), 1-11. DOI: 10.1007/ S10916-015-0326-Z

Runeson, P., \& Höst, M. (2009). Guidelines for conducting and reporting case study research in software engineering. Empirical Software Engineering, 14(2), 131-164.

SEI, S. E. (Noviembre de 2010). SEI Digital Library. Obtenido de http://www.sei.cmu. edu/reports/10tro34.pdf

Simon, J.-M., Emam, K. E., Rousseau, S., Jacquet, E., \& Babey, F. (1997). The Reliability of ISO/IEC PDTR 15504 Assessments. Software Process: Improvement and Practice, 3(3), 177-188. 